\title{
A CHARACTERIZATION OF NONUNITAL OPERATOR ALGEBRAS
}

\author{
ZHONG-JIN RUAN
}

(Communicated by Palle E. T. Jorgensen)

\begin{abstract}
We give an abstract matrix norm characterization for operator algebras with contractive approximate identities by using the second dual approach. We show that if $A$ is an $L^{\infty}$-Banach pseudoalgebra with a contractive approximate identity, then the second dual $A^{* *}$ of $A$ is a unital $L^{\infty}$-Banach pseudoalgebra containing $A$ as a subalgebra. It follows from the Blecher-RuanSinclair characterization theorem for unital operator algebras that $A^{* *}$ is completely isometrically unital isomorphic to a concrete unital operator algebra on a Hilbert space. Thus $A$ is completely isometrically isomorphic to a concrete nondegenerate operator algebra with a contractive approximate identity.
\end{abstract}

\section{INTRODUCTION}

It is well known that for any Hilbert space $H$ there is a natural matrix norm on $B(H)$, i.e., there is a natural norm $\|\cdot\|_{n}$ on each $M_{n}(B(H)) \quad(n \in \mathbb{N})$ obtained by identifying $M_{n}(B(H))$ with $B\left(H^{n}\right)$, where $H^{n}$ is the direct sum of $n$ copies of $H$. We call this family of norms $\left\{\|\cdot\|_{n}\right\}$ the operator matrix norm on $B(H)$. A norm closed subspace (resp., a norm closed subalgebra) of $B(H)$ together with the operatcr matrix norm is called a concrete operator space (resp., a concrete operator algebra). A concrete operator algebra is called unital if it contains the identity operator on the Hilbert space.

In [Ru, BRS], we have succeeded in characterizing concrete operator spaces and concrete unital operator algebras as $L^{\infty}$-matricially normed spaces and unital $L^{\infty}$-Banach pseudoalgebras (or equivalently, unital $L^{\infty}$-Banach algebras), respectively. There is no doubt that the Blecher-Ruan-Sinclair characterization theorem for unital operator algebras will be very useful in the study of nonselfadjoint operator algebras. However, many interesting algebras fail to have a unit, and there is no good characterization result for general nonunital operator algebras (cf. [BRS]).

We have found that a very important class of operator algebras, i.e., operator algebras with contractive approximate identities, can be very well characterized by their matrix norms. This is a reasonably large class of operator algebras

Received by the editors August 31, 1992.

1991 Mathematics Subject Classification. Primary 47D25; Secondary 47D15.

Key words and phrases. Operator algebras, $L^{\infty}$-Banach pseudoalgberas, $L^{\infty}$-matricially normed spaces, operator duals, completely bounded maps.

This research was partially supported by the National Science Foundation. 
since it contains all unital operator algebras and all (not necessarily unital) $C^{*}$ algebras.

In a recent joint work [PR] with Poon, we have succeeded in charaterizing these operator algebras as $L^{\infty}$-Banach algebras with contractive approximate identities. In [PR], we used the double centralizer algebra approach. The key idea is to show that, for any $L^{\infty}$-Banach algebra $A$ with a contractive approximate identity, the double centralizer algebra $M(A)$ of $A$ is completely isometrically unital isomorphic to a concrete unital operator algebra. Since the algebra $A$ is associative, $A$ can be naturally identified with a two-sided ideal of $M(A)$ and thus can be identified with a concrete operator algebra. In this approach, the associativity of the algebras is an essential condition.

In this paper, we provide a different proof of this characterization theorem by using the second dual approach. In this case, it is not necessary to assume the associativity of the algebras, i.e., we can characterize these operator algebras as " $L{ }^{\infty}$-Banach pseudoalgebras". Our main result, Theorem 2.2 , shows that if $A$ is an $L^{\infty}$-Banach pseudoalgebra with a contractive approximate identity, then the second dual $A^{* *}$ of $A$ is a unital $L^{\infty}$-Banach pseudoalgebra containing $A$ as a subalgebra. It follows from the Blecher-Ruan-Sinclair characterization theorem for unital operator algebras that $A^{* *}$ is completely isometrically unital isomorphic to a concrete unital operator algebra. Thus the subalgebra $A$ is completely isometrically isomorphic to a concrete nondegenerate operator algebra with a contractive approximate identity. We recall that a concrete operator algebra $A$ is called nondegenerate on a Hilbert space $H$ if $\overline{[A H]}$, the closure of the linear span of $A H$, is equal to $H$.

We note that, owing to Theorem 2.2 , the multiplications of these $L^{\infty}$-Banach pseudoalgebras are automatically associative if they possess contractive approximate identities. In this case, the terminologies of " $L$-Banach pseudoalgebras" and " $L$-Banach algebras" are equivalent.

\section{Preliminaries}

An $L^{\infty}$-matricially normed space is a vector space $V$ over the complex numbers $\mathbb{C}$ together with a norm $\|\cdot\|_{n}$ on each matrix space $M_{n}(V)$ such that the following conditions are satisfied:

$$
\begin{aligned}
\|x \oplus y\|_{n+m} & =\max \left\{\|x\|_{n},\|y\|_{m}\right\}, \\
\|\alpha x \beta\|_{n} & \leq\|\alpha\|\|x\|_{n}\|\beta\|
\end{aligned}
$$

for all $x \in M_{n}(V), y \in M_{m}(V)$, and $\alpha, \beta \in M_{n}(\mathbb{C})$ (cf. [Ef, Ru]). In this paper, we assume that all $L^{\infty}$-matricially normed spaces are norm complete.

Let $V, W$, and $Z$ be $L^{\infty}$-matricially normed spaces. A bilinear map $m: V \times W \rightarrow Z$ is called completely contractive if

$$
m_{n}\left(\left[a_{i j}\right],\left[b_{j k}\right]\right)=\left[\sum_{j=1}^{n} m\left(a_{i j}, b_{j k}\right)\right]
$$

satisfies

$$
\left\|m_{n}\left(\left[a_{i j}\right],\left[b_{j k}\right]\right)\right\|_{n} \leq\left\|\left[a_{i j}\right]\right\|_{n}\left\|\left[b_{j k}\right]\right\|_{n}
$$

for all $\left[a_{i j}\right] \in M_{n}(V),\left[b_{j k}\right] \in M_{n}(W)$, and $n \in \mathbb{N}$. 
An $L^{\infty}$-Banach pseudoalgebra is a vector space $A$ over the complex number $\mathbb{C}$ such that

(1) $A$ is an $L^{\infty}$-matricially normed space,

(2) there is a completely contractive bilinear map $m: A \times A \rightarrow A$.

The completely contractive bilinear map $m$ is called the pseudomultiplication of $A$. An $L^{\infty}$-Banach pseudoalgebra $A$ is called unital if $A$ contains a distinguished element $e$ of norm one such that

(3) $m(a, e)=a=m(e, a)$

for all $a \in A$. An $L^{\infty}$-Banach pseudoalgebra $A$ is said to have a contractive approximate identity if there is a net $\left\{a_{\alpha}: \alpha \in \Lambda\right\}$ of contractive elements in $A$ such that

$\left(3^{\prime}\right) \quad\left\|m\left(a_{\alpha}, a\right)-a\right\| \rightarrow 0$ and $\left\|m\left(a, a_{\alpha}\right)-a\right\| \rightarrow 0$

for all $a \in A$.

It is easy to see that every concrete unital operator algebra (resp., every concrete operator algebra with a contractive approximate identity) is a unital $L^{\infty}$ Banach pseudoalgebra (resp., an $L^{\infty}$-Banach pseudoalgebra with a contractive approximate identity). On the other hand, we have

Theorem 2.1 [BRS]. Every unital $L^{\infty}$-Banach pseudoalgebra is completely isometrically unital isomorphic to a concrete unital operator algebra.

Theorem 2.2 (Main Theorem). Every $L^{\infty}$-Banach pseudoalgebra with a contractive approximate identity is completely isometrically isomorphic to a concrete nondegenerate operator algebra with a contractive approximate identity.

\section{THE PROOF OF THEOREM 2.2}

Let $A$ be an $L^{\infty}$-Banach pseudoalgebra with a contractive approximate identity $\left\{a_{\alpha}\right\}$, and let $m: A \times A \rightarrow A$ be the completely contractive pseudomultiplication of $A$. In this section, we give a proof of Theorem 2.2 by using the second dual approach. The key point is to show that the bilinear map $m$ can be extended to a (unique) normal completely contractive bilinear map $\bar{m}: A^{* *} \times A^{* *} \rightarrow A^{* *}$, which satisfies the following condition: for any $x^{* *}, y^{* *} \in A^{* *}$, we have

$$
\lim _{\alpha} \lim _{\beta} m\left(x_{\alpha}, y_{\beta}\right)=\bar{m}\left(x^{* *}, y^{* *}\right)=\lim _{\beta} \lim _{\alpha} m\left(x_{\alpha}, y_{\beta}\right),
$$

where $\left\{x_{\alpha}\right\}$ and $\left\{y_{\beta}\right\}$ are nets in $A$ such that $x_{\alpha} \rightarrow x^{* *}$ and $y_{\beta} \rightarrow y^{* *}$ in the $\sigma\left(A^{* *}, A^{*}\right)$-topology, respectively. This implies that the extended bilinear map $\bar{m}$ is equal to the left (resp., right) Arens's extension of $m$ and thus the bilinear map $m$ is Arens's regular (cf. [Ar]). We carry out the proof in the following steps:

(1) Since $A$ is an $L^{\infty}$-matricially normed space, it is completely isometrically linear isomorphic to a concrete operator space (cf. [Ru]). Thus $A$ can be identified with a norm closed subspace of a $C^{*}$-algebra $B$. Assume that the $C^{*}$-algebra $B$ is acting universally on a Hilbert space $H$, i.e., regarding (id, $H$ ) as the universal representation of $B$. Then $B^{* *}$, the second dual of $B$, can be identified with $\bar{B}^{\sigma}$, the $\sigma$-weak closure of $B$ in $B(H)$. It follows that $A^{* *}$, the second dual of $A$, can be identified with $\bar{A}^{\sigma}$, the $\sigma$-weak closure of $A$ in 
$B(H)$. In this case, the $\sigma\left(A^{* *}, A^{*}\right)$-topology on $A^{* *}$ coincides with the $\sigma$-weak topology on $\bar{A}^{\sigma}$.

(2) The completely contractive bilinear map $m: A \times A \rightarrow A \subseteq B(H)$ can be extended to a completely contractive bilinear map $m^{\prime}: B \times B \rightarrow$ $B(H)$ (cf. [CS, PS]). Furthermore, there exist Hilbert spaces $K_{1}$ and $K_{2}$, *-representations $\pi_{i}: B \rightarrow B\left(K_{i}\right)$, and contractive linear operators $V_{1} \in$ $B\left(K_{1}, H\right), T \in B\left(K_{2}, K_{1}\right)$, and $V_{2} \in B\left(H, K_{2}\right)$ such that

$$
m^{\prime}(x, y)=V_{1} \pi_{1}(x) T \pi_{2}(y) V_{2}
$$

for all $x, y \in B$.

(3) It is well known that the *-representations $\pi_{i}$ in (3.1) can be uniquely extended to $\sigma$-weakly continuous ${ }^{*}$-representations $\bar{\pi}_{i}: B^{* *} \rightarrow B\left(K_{i}\right)$. Thus the completely contractive bilinear map $m^{\prime}$ can be naturally extended to a normal completely contractive bilinear map $\bar{m}: B^{* *} \times B^{* *} \rightarrow B(H)$ given by

$$
\bar{m}\left(x^{* *}, y^{* *}\right)=V_{1} \bar{\pi}_{1}\left(x^{* *}\right) T \bar{\pi}_{2}\left(y^{* *}\right) V_{2}
$$

for all $x^{* *}, y^{* *} \in B^{* *}$. The terminology "normal" means that $\bar{m}$ is separately $\sigma$-weakly continuous in each variable. Restricted to $A^{* *}$, we get a normal completely contractive bilinear map $\bar{m}: A^{* *} \times A^{* *} \rightarrow B(H)$.

(4) First we claim that $\bar{m}$ maps $A^{* *} \times A^{* *}$ into $A^{* *}$. To see this, let $x^{* *}$ and $y^{* *}$ be two arbitrary elements in $A^{* *}$. Then there exist two nets $\left\{x_{\alpha}\right\}$ and $\left\{y_{\beta}\right\}$ in $A$ with $\left\|x_{\alpha}\right\| \leq\left\|x^{* *}\right\|$ and $\left\|y_{\beta}\right\| \leq\left\|y^{* *}\right\|$ such that $x_{\alpha} \rightarrow x^{* *}$ and $y_{\beta} \rightarrow y^{* *}$ in the $\sigma$-weak topology, respectively. Fixing $x_{\alpha}$ and taking the limit on $y_{\beta}$, we get

$$
\bar{m}\left(x_{\alpha}, y^{* *}\right)=\lim _{\beta} \bar{m}\left(x_{\alpha}, y_{\beta}\right)=\lim _{\beta} m\left(x_{\alpha}, y_{\beta}\right) \in \bar{A}^{\sigma}=A^{* *} .
$$

Then fixing $y^{* *}$ and taking the limit on $x_{\alpha}$, we get

$$
\bar{m}\left(x^{* *}, y^{* *}\right)=\lim _{\alpha} \bar{m}\left(x_{\alpha}, y^{* *}\right) \in \bar{A}^{\sigma}=A^{* *} .
$$

This proves the claim.

We note that this also shows that the extended bilinear map $\bar{m}$ is equal to the left Arens's extension of $m$, i.e.,

$$
\bar{m}\left(x^{* *}, y^{* *}\right)=\lim _{\alpha} \lim _{\beta} m\left(x_{\alpha}, y_{\beta}\right) .
$$

By exchanging the order of the limits in the above argument, one can show that $\bar{m}$ is also equal to the right Arens's extension of $m$, i.e.,

$$
\bar{m}\left(x^{* *}, y^{* *}\right)=\lim _{\beta} \lim _{\alpha} m\left(x_{\alpha}, y_{\beta}\right) .
$$

Thus the bilinear map $m$ is Arens's regular (cf. [Ar]).

Finally we note that since $\bar{m}$ is normal from $A^{* *} \times A^{* *}$ into $A^{* *}$ and $A$ is $\sigma$-weakly dense in $A^{* *}, \bar{m}$ is a unique such extension of $m$.

(5) Since $A$ is an $L^{\infty}$-matricially normed space, there is a natural $L^{\infty}$. matricial norm structure on $A^{*}$ by identifying $M_{n}\left(A^{*}\right)$ with $C B\left(A, M_{n}\right)$, the space of all completely bounded maps from $A$ into $M_{n}$ (cf. [BP, ER]). $A^{*}$ with this matrix norm is called the operator dual of $A$. If we consider $A^{* *}$ as the operator second dual of $A$, then $A^{* *}$ is an $L^{\infty}$-matricially normed space 
and the extended bilinear map $\bar{m}: A^{* *} \times A^{* *} \rightarrow A^{* *}$ is completely contractive. Thus $\left(A^{* *}, \bar{m}\right)$ is an $L^{\infty}$-Banach pseudoalgebra.

Since $A$ has a contractive approximate identity $\left\{a_{\alpha}\right\}$, by passing to a subnet if it is necessary, we can assume that the $\left\{a_{\alpha}\right\}$ converges to an element $e \in A^{* *}$ in the $\sigma$-weak topology. From (3.3) and (3.4), it is easy to see that $e$ is an element of norm one and

$$
\bar{m}\left(x^{* *}, e\right)=x^{* *}=\bar{m}\left(e, x^{* *}\right)
$$

for all $x^{* *} \in A^{* *}$. Thus $A^{* *}$ is a unital $L^{\infty}$-Banach pseudoalgebra. It follows from Theorem 2.1 that $A^{* *}$ is completely isometrically unital isomorphic to a concrete unital operator algebra.

Since the canonical embedding map $A \rightarrow A^{* *}$ is a complete isometry (cf. [Bl]) and $\bar{m}=m$ when restricted to $A, A$ can be identified with a subalgebra of $A^{* *}$. This shows that $A$ is completely isometrically isomorphic to a concrete operator algebra with a contractive approximate identity.

(6) It follows from (5) that $A$ is completely isometrically isomorphic to a concrete operator algebra. Thus $A^{* *}$ can be identified with a $\sigma$-weakly closed subalgebra of bounded linear operators on a Hilbert space $H$. Since $A^{* *}$ has a unit $e$, one can assume, without loss of generality, that $e=1_{H}$, the identity operator on $H$. Since $A$ is $\sigma$-weakly dense in $A^{* *}$, the contractive approximate identity $\left\{a_{\alpha}\right\}$ of $A$ converges $\sigma$-weakly, and thus weakly, to $1_{H}$. It follows that $A$ is nondegenerate on $H$. This completes the proof.

To close this section, we make the following remark.

Let $A$ be an operator algebra with a contractive approximate identity. If $A$ has a unit, we can identify the unit of $A$ with the unit of $A^{* *}$. If $A$ has no unit, we let $A^{1}=A \oplus \mathbb{C}$ be the unitalization of $A$ with the multiplication defined by

$$
(a, \alpha) \cdot(b, \beta)=(a \cdot b+\alpha b+\beta a, \alpha \beta)
$$

for all $(a, \alpha),(b, \beta) \in A^{1}$. Then we can identify $A^{1}$ with a unital subalgebra of $A^{* *}$ and get an operator algebra matrix norm on $A^{1}$. It turns out that this is the only operator algebra matrix norm on $A^{1}$ (cf. [PR, §5]). Furthermore, if $A$ and $B$ are two operator algebras with contractive approximate identities, then every completely isometric isomorphism from $A$ onto $B$ can be uniquely extended to a completely isometric unital isomorphism from $A^{1}$ onto $B^{1}$. Therefore, in many cases, one can reduce the nonunital problems to unital ones.

\section{REFERENCES}

[Ar] R. Arens, The adjoint of a bilinear operation, Proc. Amer. Math. Soc. 2 (1951), 839-848.

[Bl] D. Blecher, The standard dual of an operator space, Pacific J. Math. 153 (1992), 15-30.

[BP] D. Blecher and V. Paulsen, Tensor products of operator spaces, J. Funct. Anal. 99 (1991), 262-292.

[BRS] D. Blecher, Z.-J. Ruan, and A. Sinclair, A characterization of operator algebras, J. Funct. Anal. 89 (1990), 188-201.

[CS] E. Christensen and A. Sinclair, Representations of completely bounded multilinear operators, J. Funct. Anal. 72 (1987), 151-181.

[Ef] E. G. Effros, Advinces in quantized functional analysis, Proceedings of ICM (Berkeley, 1986), Amer. Math. Soc., Providence, RI, 1987, pp. 906-916. 
[ER] E. G. Effros and Z.-J. Ruan, $A$ new approach to operator spaces, Bull. Canad. Math. Soc. 34 (1991), 329-337.

[PS] V. I. Paulsen and R. R. Smith, Multilinear maps and tensor norms on operator systems, J. Funct. Anal. 73 (1987), 258-276.

[PR] Y. T. Poon and Z.-J. Ruan, Operator algebras with contractive approximate identities, Canad. J. Math. (to appear).

[Ru] Z.-J. Ruan, Subspaces of $C^{*}$-algebras, J. Funct. Anal. 76 (1988), 217-230.

Department of Mathematics, University of Illinois, Urbana-Champaign, Urbana, IlliNoIs 61801

E-mail address: ruanesymcom.math.uiuc.edu 